\title{
Condensation of helium in nanoscopic alkali wedges at zero temperature
}

\author{
E. S. Hernández \\ Departamento de Física, Facultad de Ciencias Exactas y Naturales, Universidad de Buenos Aires, \\ and Consejo Nacional de Investigaciones Científicas y Técnicas, Buenos Aires, Argentina \\ F. Ancilotto \\ Dipartimento di Fisica 'G. Galilei', Università di Padova, via Marzolo 8, I-35131 Padova, Italy, \\ and DEMOCRITOS National Simulation Center, Trieste, Italy \\ M. Barranco, R. Mayol, and M. Pi \\ Departament ECM, Facultat de Física, Universitat de Barcelona, 08028 Barcelona, Spain \\ (Received 27 February 2006; revised manuscript received 25 April 2006; published 6 June 2006)
}

\begin{abstract}
We present a complete calculation of the structure of liquid ${ }^{4} \mathrm{He}$ confined to a concave nanoscopic wedge, as a function of the opening angle of the walls. This is achieved within a finite-range density functional formalism. The results here presented, restricted to alkali metal substrates, illustrate the change in meniscus shape from rather broad to narrow wedges on weak and strong alkali adsorbers, and we relate this change to the wetting behavior of helium on the corresponding planar substrate. As the wedge angle is varied, we find a sequence of stable states that, in the case of cesium, undergo one filling and one emptying transition at large and small openings, respectively. A computationally unambiguous criterion to determine the contact angle of ${ }^{4} \mathrm{He}$ on cesium is also proposed.
\end{abstract}

DOI: 10.1103/PhysRevB.73.245406

\section{INTRODUCTION}

The current possibility of tailoring the production of adsorbing surfaces in the nanoscopic scale raises a series of questions on the role of inhomogeneities in the growth of liquid films on nanopatterned planar substrates. In particular, the wedge geometry is a simple one that permitted one to carry on many theoretical anticipations of the wetting behavior of fluids in such confinement (see, e.g., Refs. 1-3 and cited therein), and it has been recently argued ${ }^{3}$ that models of fluids adsorbed in planar wedges can be regarded as a generic class of structured inhomogeneous fluids.

Since the early calculation of physisorption of noble gases in an oblique corner, ${ }^{4}$ most predictions of the shape changes undergone by the liquid meniscus are based on the examination of the equilibrium liquid-vapor interface of a macroscopic sample at constant bulk density $\rho_{0}$, whose sharp free surface is described by a function determined by minimization of the surface free energy. The latter contains the energy cost for building a curved liquid-vapor interface with surface tension $\sigma_{l v}$, in addition to the interfacial contributions governed by the surface energies $\sigma_{s l}$ and $\sigma_{s v}$, with $l, s, v$ denoting liquid, solid, and vapor/vacuum, respectively. These three surface tensions determine the contact angle $\theta$ as given by the classical Young's relation. ${ }^{5-7}$ These calculations confirm the thermodynamic theory of wetting in a wedge ${ }^{8}$ that shows, on the ground of purely macroscopic arguments, that a filling transition characterized by a perfectly planar liquidvapor interface takes place, at liquid-vapor coexistence, when the opening angle $2 \varphi$ of the walls is related to the contact angle for a planar surface as $\varphi=\pi / 2-\theta$. Moreover, the above theories classify the meniscus shapes for nonwetting liquids according to the following sequence:6,7

(1) $\theta>\frac{\pi}{2}+\varphi$, spherical bridge;

(2) $\frac{\pi}{2}+\varphi \geqslant \theta>\frac{\pi}{2}-\varphi$, spherical convex meniscus;
PACS number(s): 67.70.+n, 64.90.+b, 68.03. $-\mathrm{g}, 68.08 . \mathrm{Bc}$

(3) $\theta=\frac{\pi}{2}-\varphi$, planar meniscus;

(4) $\theta<\frac{\pi}{2}-\varphi$, spherical concave meniscus.

While classical, macroscopic approaches provide a solid frame for the expected behavior of fluids in wedges, one may wonder if these predicted phenomena are robust at the nanoscale. A study of interfacial phenomena in power-law wedges permits one to trace the route from wetting to capillary condensation $^{2}$ and suggests the possibility of adapting the adsorptive abilities of solid substrates by shaping its surface inhomogeneities at the nanoscopic level. Recent measurements of the growth of Ar films on an array of microscopic linear wedges ${ }^{9}$ confirm this conjecture and demonstrate a clear crossover between a planarlike and a geometrydependent behavior. On the theoretical side, it has been remarked $^{7}$ that the full one-body density $\rho(\mathbf{r})$ for the confined particles should be obtained either from simulations or from density functional (DF) theory. With the latter instrument, we have recently investigated the growth of nanoscopic ${ }^{4} \mathrm{He}$ clusters on planar alkali surfaces at zero temperature $(T)$, employing a finite range $\mathrm{DF} \cdot{ }^{10}$ The analysis of the energetics and shape systematics with increasing number of atoms $N$ shows that helium drops on wettable alkali surfaces reach a maximum vertical height equal to the thickness of the prewetting film, with the chemical potential at the prewetting jump in the zero temperature adsorption isotherm. These results illustrate that DF-based calculations can account for the interplay between geometry and thermodynamics in nanoscopic systems, sufficiently large to remain beyond the reach of present microscopic many-body theories. On these theoretical grounds, in this work we carry out a systematic investigation of the spatial structure and shape evolution of nanoscopic helium samples in linear alkali wedges, as described in the next sections. In particular, in Sec. II we shortly review the DF formalism employed, and in $\mathrm{Sec}$. III we present our results for $\mathrm{Cs}$ and $\mathrm{Na}$ wedges, which are summarized in Sec. IV. 


\section{METHOD}

Our geometry is translationally invariant along one spatial direction ( $y$ axis). The equilibrium ${ }^{4} \mathrm{He}$ density profile $\rho(x, z)$ will thus depend on the $(x, z)$ coordinates only. We compute the density profile that minimizes the zero temperature grand potential $\Omega=E-\mu N$, with $\mu$ the chemical potential of the helium atoms and with $N$ the grand canonical ensemble average of the particle number operator, that in a wedge extending a macroscopic length $L$ along the $y$ direction, takes the form

$$
N=\int d^{3} \mathbf{r} \rho(\mathbf{r}) \equiv L \iint d x d z \rho(x, z)
$$

The variation $\delta \Omega / \delta \rho$ gives rise to the integrodifferential Euler-Lagrange equation

$$
\left[-\frac{\hbar^{2}}{2 m} \nabla^{2}+V(\rho)+V_{s}(x, z)\right] \sqrt{\rho(x, z)}=\mu \sqrt{\rho(x, z)} .
$$

Here $V(\rho)$ is the effective potential arising from functional differentiation of the potential energy density per unit length, and $V_{s}(x, z)$ is the wedge potential. In this work, we have selected the full Orsay-Trento (OT) DF, ${ }^{11}$ which is known to provide a quite accurate description of inhomogeneous structures of ${ }^{4} \mathrm{He}$ at $T=0$. We can safely neglect gravitational effects in our calculations, since the capillary length $a$ $=\left[2 \sigma_{l v} /\left(g m \rho_{0}\right)\right]^{1 / 2}$, with $\rho_{0}=0.0218 \AA^{-3}$ the saturation density of ${ }^{4} \mathrm{He}$ and $g$ the acceleration of gravity, is much larger than any linear dimension of the ${ }^{4} \mathrm{He}$ samples considered in the present work.

To our knowledge, the best available adsorbing potential for helium on alkali planar surfaces is the Chizmeshya-ColeZaremba (CCZ) potential. ${ }^{12,13}$ As a compromise between rigor and physical insight, ${ }^{14}$ in the present work we have chosen to approximate $V_{s}(x, z)$ by the summation of two planar CCZ potentials at an angle $2 \varphi$. Double-counting effects, even when sizeable, are only important for fairly large values of $2 \varphi$ and in the vicinity of the wedge apex. ${ }^{3}$ Thus, one should not expect qualitative differences in the density patterns for the fairly large linear densities considered here.

Equation (2) is discretized with 13-point formulas for the partial derivatives and solved by an imaginary time method, ${ }^{10}$ employing a fast Fourier transform algorithm ${ }^{15}$ to obtain $V(\rho)$ from the atom density $\rho(x, z) .{ }^{16}$ We have verified that the numerical outcome is stable against the increase of the number of mesh points and the order of the discretization formulas.

\section{RESULTS}

\section{A. Cesium wedges}

We address first the most interesting case of cesium, which is not wetted by ${ }^{4} \mathrm{He}$ at $T=0$. For reference, we have computed a sequence of ${ }^{4} \mathrm{He}$ pancake-shaped systems on a flat Cs surface, namely, translationally invariant systems in the $y$ direction and characterized by the linear density $n$ $=N / L$. Figure 1 shows the chemical potential of the ${ }^{4} \mathrm{He}$ atoms as a function of $n$, that displays a neat tendency to-

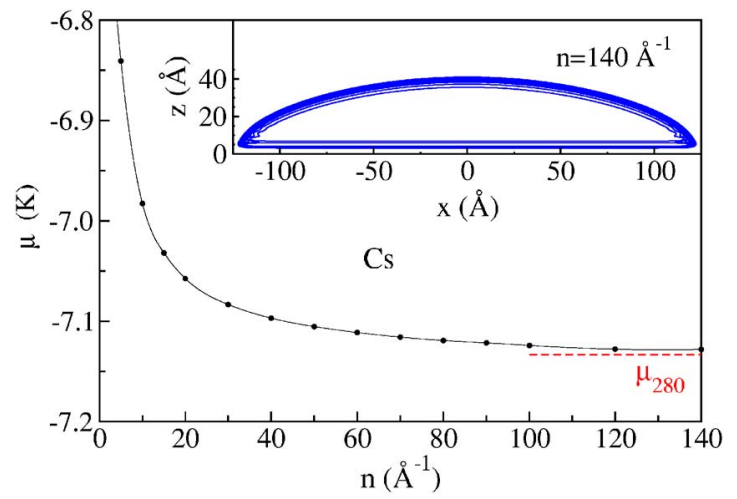

FIG. 1. (Color online) Chemical potential of a ${ }^{4} \mathrm{He}$ pancake on planar Cs as a function of linear density. The chemical potential of the $n=280 \AA^{-1}$ pancake is indicated by a horizontal dashed line. The inset shows, for the $n=140 \AA^{-1}$ density profile, the equidensity lines from $\rho=0.9 \rho_{0}$ to $0.1 \rho_{0}$ in $0.1 \rho_{0}$ steps.

wards saturation at the bulk figure $\mu_{0}=-7.15 \mathrm{~K}$. The equidensity lines for the density profile corresponding to a linear density $n=140 \AA^{-1}$ are shown in the inset of this figure. The profiles are qualitatively similar to those shown in Ref. 10 in the case of the deposited drops, and confirm the nanoscopic spatial scale of the systems.

It should be kept in mind that in a typical adsorption experiment, one changes the chemical potential by varying the pressure in the chamber towards saturated vapor pressure (SVP) conditions. Very close to SVP (but below it) the thermodynamic equilibrium state of liquid ${ }^{4} \mathrm{He}$ on $\mathrm{Cs}$ is a thin, microscopic planar film partially wetting the surface. Nonetheless macroscopic, metastable ${ }^{4} \mathrm{He}$ droplets can be realized if a finite amount of helium is deposited on the Cs surface, as shown for instance in the experimental results of Ref. 19. The contact angle of such droplets with the surface plane is a meaningful quantity to be measured/calculated and which can be related to thermodynamic, equilibrium, properties. We shall see in the following that similar nonequilibrium configurations are found in the wedge geometry, which might be experimentally realized under particular conditions.

When helium at given $n$ is placed in a Cs wedge and the opening angle $2 \varphi$ is varied, we generate the shape sequence illustrated in Fig. 2, at a linear density of $280 \AA^{-1}$. This evolution illustrates the modifications of the wetting behavior of helium on Cs due to the confinement exerted by the corner. A convex helium pancake such as the one shown in the inset of Fig. 1 initially adsorbed on a flat Cs surface, when subjected to the formation of a wedge and decrease of the opening angle experiences a sequence of changes whose highlights are: (a) a filling transition where the curvature vanishes, for an opening near $110^{\circ}$; (b) formation of a concave meniscus that persists within a sizable range of angles; (c) an "emptying" transition where a flat meniscus appears near $52^{\circ}$, with restoration of the convex shape for smaller angles; (d) formation of a bridge that will be expelled from the wedge for a sufficiently small angle. We have verified that the bridge profiles are perfectly circular, as predicted by the classical theories. ${ }^{4-7}$ Note, however, that here the term "transition" does not address a thermodynamic phase transi- 


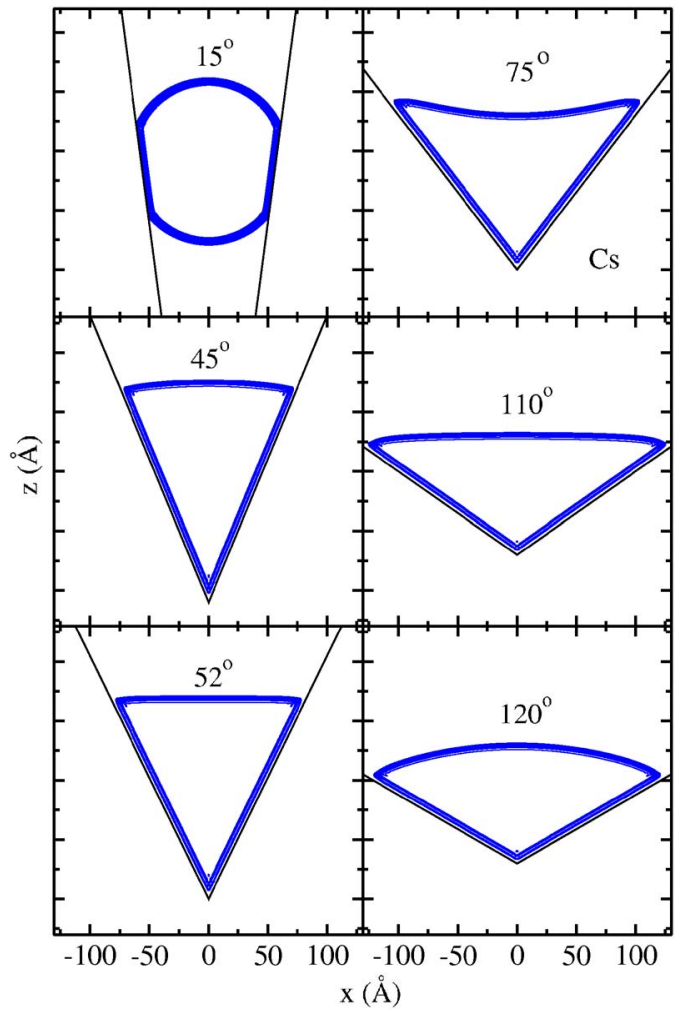

FIG. 2. (Color online) Density profiles of helium at $n$ $=280 \AA^{-1}$ in a Cs corner represented by thin straight lines, for angular opening $2 \varphi=15,45,52,75,110$, and $120^{\circ}$. The distance between major tick marks on the $z$ axis is $50 \AA$. The center of the $15^{\circ}$ configuration is about $400 \AA$ above the apex of the wedge. Equidensity lines are drawn as in Fig. 1.

tion, but a crossover between two different, well defined regimes characterized by geometry and energetics as analyzed in what follows.

The above sequence can be understood by examining the energetics of helium atoms at several linear densities, as functions of the wedge opening. For this sake, in Fig. 3 we plot the chemical potential for $n=70,140$, and $280 \AA^{-1}$. Several interesting features become evident. First, the three curves cross at the bulk value $\mu_{0}$ for two opening angles, namely the filling one $2 \varphi_{F}=110^{\circ}$ and the emptying one $2 \varphi_{E}=52^{\circ}$. Within this interval, the helium chemical potential $\mu$ lies below the thermodynamic limit $\mu_{0}$, and the systems are thermodynamically stable, since $d \mu / d n$ is positive. By contrast, above the filling angle $\varphi_{F}$ and below the emptying angle $\varphi_{E}, \mu$ lies above the bulk value with negative $d \mu / d n$; the corresponding helium samples are then metastable. These characteristics can be further analyzed looking at the grandpotential per unit length $\Omega / L=E / L-\mu n$, where $E / L$ is the energy per unit length, displayed in Fig. 4 for the same linear densities. Note that in the present context, $\Omega / L$ represents the surface-plus-line energy difference per unit length between a wedge filled with liquid and an empty wedge. ${ }^{7}$ At $\varphi_{F}, \Omega / L$ is independent of $n$ and vanishes for these large, however nanoscopic, amounts of fluid. Thus, the conditions for a filling transition, previously derived for macroscopic systems, ${ }^{7}$ are fulfilled for these helium samples. This suggests an effective way of determining the contact angle $\theta$ of

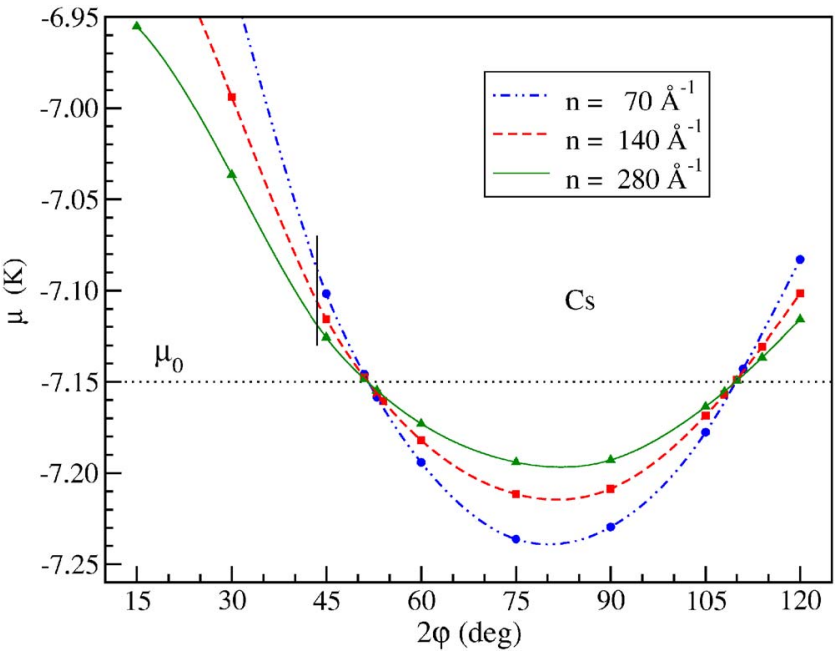

FIG. 3. (Color online) Chemical potential of helium atoms in a Cs wedge as a function of angular opening $2 \varphi$ (degrees) for linear densities 70,140 , and $280 \AA^{-1}$. Wedge configurations to the left of the thin vertical segment at about $43^{\circ}$ have higher energies than a pancake, with the same linear density, adsorbed on a planar surface.

${ }^{4} \mathrm{He}$ on Cs by means of DF calculations; we recall that previous DF-based calculations of this angle give values between $31^{\circ}$ (Ref. 17) and $36^{\circ},{ }^{16}$ in good agreement with experiments in Refs. 18 and 19. Other DF estimates provide somewhat smaller values,${ }^{20}$ whereas a different experimental determination of the contact angle ${ }^{21}$ yields $\theta=48^{\circ} \pm 1^{\circ}$. Using the value of $2 \varphi_{F} \sim 110^{\circ}$ where the curves cross in Fig. 4 we obtain here a contact angle $\theta=\pi / 2-\varphi_{F}=35^{\circ}$.

Figure 4 also shows, in correspondence with Fig. 3, that a second curve crossing, where $\Omega / L$ is $n$ independent, occurs at the aperture $2 \varphi_{E} \sim 53^{\circ}$ i.e., at the threshold for the formation of the bridge, which we have found to be clearly visible only below $\sim 30^{\circ}$. Except in a very narrow angular region near $\varphi_{E}$, whose existence is likely attributable to finite size effects, ${ }^{22} \Omega / L$ is negative in the interval $\left[\varphi_{E}, \varphi_{F}\right]$, indicating that condensation might occur either in a continuous way or

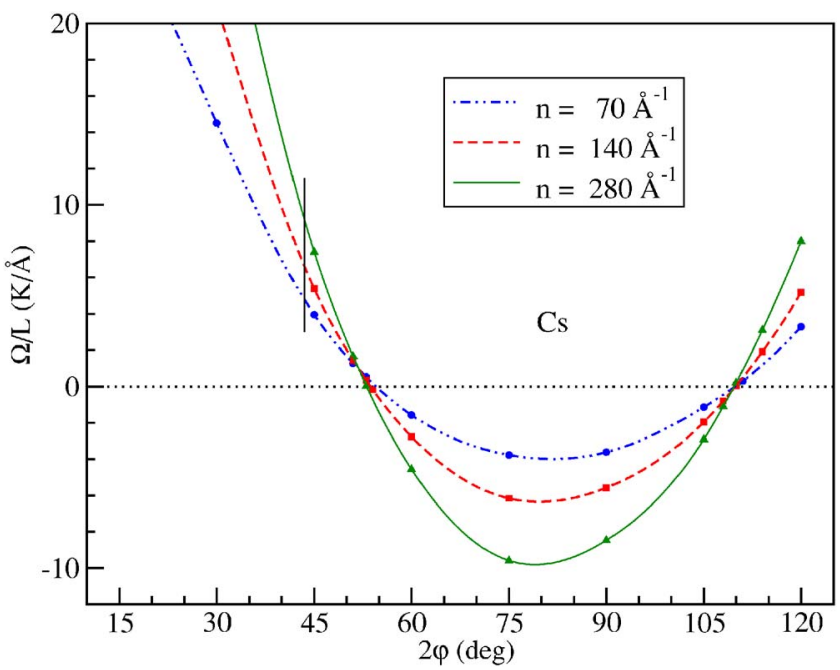

FIG. 4. (Color online) Same as Fig. 3 for the grand potential per unit length. 


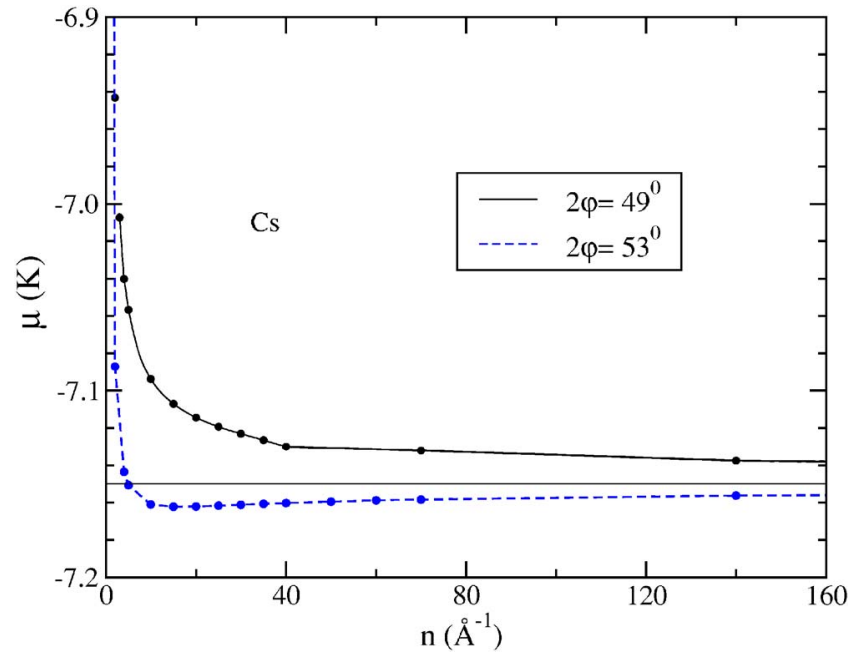

FIG. 5. (Color online) Chemical potential of helium atoms in a Cs wedge as a function of linear densities. Solid line: $2 \varphi=49^{\circ}$; dashed line: $2 \varphi=53^{\circ}$.

through discontinuous jumps in the linear coverage. In fact, the latter is the case here, as shown below in relation to Fig. 5.

Within the interval $\left[\varphi_{E}, \varphi_{F}\right]$, the gain in energy originates in the disappearance of the solid-vacuum interface at the meniscus contact line, that leaves only a liquid-vacuum interface at the free surface, as seen in Fig. 2. This is reversed for $\varphi<\varphi_{E}$ since the system shifts to a nonwetting regime. In these wedges, the energy balance favors the presence of vacuum, rather than liquid, at the corner, making room for the formation of the inner convex meniscus that "dries" the corner and forms the bridge, at the price of increasing the total energy.

The existence of two stable solutions in the $\left[\varphi_{E}, \varphi_{F}\right]$ interval for a given coverage as seen in Fig. 3, and in flat meniscus configurations at $\mu=\mu_{0}$, is a genuine nanoscopic effect, as it does not appear for macroscopic samples, where only a filling transition is predicted for liquid in a wedge made of partially wettable surfaces. ${ }^{7}$ As a matter of fact, all equilibrium macroscopic configurations, irrespective of the opening, might be thought of as corresponding to the same chemical potential, that of bulk liquid, with the fluid density in the wedge taking its value at bulk liquid-vacuum coexistence at zero temperature.

To illustrate the mechanism of condensation, in Fig. 5 we plot two selected adsorption isotherms $\mu(n)$, for angles $2 \varphi$ $=49$ and $53^{\circ}$. The respective $n=0$ ordinates - not shown in the current scale-correspond to the binding energy of a single helium atom to the wedge at each aperture, computed separately. The solid line $\left(2 \varphi=49^{\circ}\right)$ represents $\mu(n)$ for an opening slightly below $\varphi_{E}$ : the ${ }^{4} \mathrm{He}$ configurations in such wedge are always metastable with respect to the bulk, since $\mu(n)$ approaches $\mu_{0}$ from above. Thus, no condensation can occur in the wedge at such an aperture. By contrast, when the angle is slightly larger than $\varphi_{E}$ (dashed line, $2 \varphi=53^{\circ}$ ), condensation takes place with a jump in the linear density from $n=0$ to a finite value. The associated "pre-emptying" transitions occur at a value of the chemical potential which can be found by using the usual equal-area Maxwell construction [or, equivalently, by identifying the crossing between the $\mu(n)$ and $E / N$ curves $\left.^{10}\right]$. From this construction we find that at $2 \varphi=53^{\circ}$ a jump from $n=0$ to $n \sim 270 \AA^{-1}$ occurs at $\mu=$ $-7.155 \mathrm{~K}$, i.e. just below SVP. This indicates that a crossover between a filled and an empty wedge is only meaningful for linear densities near to and above $\sim 270 \AA^{-1}$, i.e., more dilute helium vapors cannot condense in the wedge at angles equal to and slightly above $2 \varphi_{E}$. A similar behavior (i.e. finite jumps in coverage below SVP) is encountered for all angles in the range $\left[2 \varphi_{E}, 2 \varphi_{F}\right]$; however, for the largest openings in this interval, condensation occurs at rather low coverages, say $n \approx 5-10 \AA^{-1}$.

The occurrence of an "emptying" regime at lower opening angle, as suggested by our results, is surprising at first sight. No emptying transition is predicted from macroscopic thermodynamic arguments, and this behavior seems also to contradict the expectation that a narrow wedge should be filled by capillary condensation (CC), below SVP, in the same way as small pores/slits are known, from theoretical calculations, to undergo a CC transition even for weakly adsorbing surfaces. $^{23,24}$ We believe that the wedge geometry makes here an important difference with respect to pore/slit case, even in the narrowest wedge, the distance between the walls can be arbitrarily large sufficiently far from the apex.

The analysis of the energy per particle for fixed $n$, as a function of wedge opening, indicates that for the linear densities under consideration, below $2 \varphi \approx 43^{\circ}$ helium bridges are metastable (i.e., have higher energies) with respect to helium pancakes deposited on planar Cs surfaces. This limit is shown in Fig. 4 by a vertical segment. Similarly, wedges corresponding to openings $2 \varphi \gtrsim 120^{\circ}$ are also metastable with respect to pancakes with the same number of atoms. In these regions (below $2 \varphi \approx 43^{\circ}$ and above $2 \varphi \gtrsim 120^{\circ}$ ) the pancake, which is a symmetry breaking configuration, is more stable than the axially symmetric configuration. It should be remarked that these metastable configurations appear for finite average numbers of ${ }^{4} \mathrm{He}$ atoms (per unit length) within a grand canonical description; as a reference, we recall that a droplet with finite atom number $N$ on $\mathrm{Cs}$ is metastable, not only with respect to bulk, but also with respect to a thin film at finite coverage $N / A$. In view of these results, we are confident that in a hypothetical experimental setup where finite, nanoscopic amounts of $\mathrm{He}$ are deposited in Cs alkali wedges, our prediction that narrow wegdes are either empty or hosting "bridge" configurations could in principle be checked.

\section{B. Sodium wedges}

We have carried out similar calculations for helium on sodium, analyzing the evolution of helium initially placed in the unstable regime at low coverages, i.e., below the prewetting transition. Figure 6 displays the chemical potential of ${ }^{4} \mathrm{He}$ pancakes on a flat $\mathrm{Na}$ surface. For this strong adsorber, the corresponding asymptotic limit is $-8.75 \mathrm{~K}$, the value of the chemical potential at the prewetting jump. ${ }^{10}$

Figures 7 and 8 display, respectively, the chemical potential and grand potential per unit length of a ${ }^{4} \mathrm{He}$ sample with 


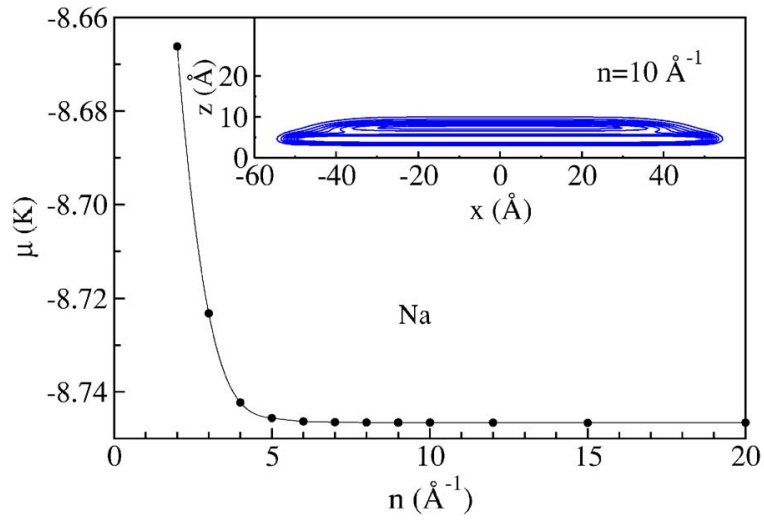

FIG. 6. (Color online) Same as Fig. 1 for ${ }^{4} \mathrm{He}$ on planar Na. In this case, the inset corresponds to $n=10 \AA^{-1}$.

$n=15 \AA^{-1}$ and shows that the filling transition is obviously absent due to the fact that sodium is wetted by ${ }^{4} \mathrm{He}$ at $T=0$. In these figures, the thresholds for the stability of pancake structures are shown by the short vertical segments.

Finally, typical density patterns are displayed in Fig. 9 for the linear density $n=15 \AA^{-1}$ and several wedge openings. The panels show the spread of the wetting liquid, that retains a rather deep concave meniscus. This figure shows that helium originally deposited on a flat, strongly wettable adsorber like Na just follows the folding of the surface with angle $2 \varphi$ and forms a deep meniscus. For sufficiently small angles the pattern evolves into a nanoscopic bridge, similar to that shown in the first panel of Fig. 2. Such configurations have, however, higher energies than the corresponding pancake structures with the same linear density, deposited on one of the walls, and thus should not occur in sodium or any other wettable wedge.

As a final remark, we can safely state that for both alkalis, the curvature of the concave meniscus changes between the

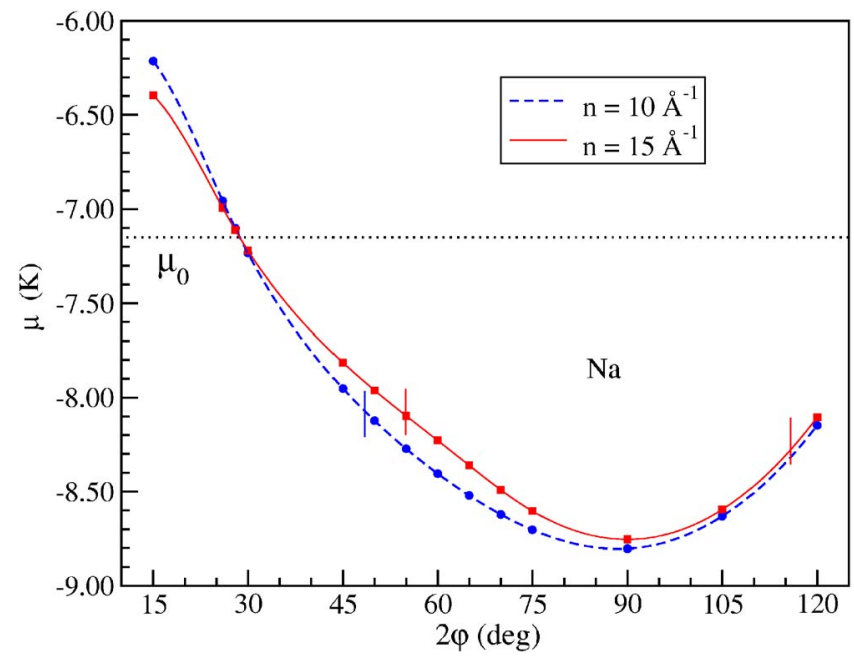

FIG. 7. (Color online) Chemical potential of helium atoms in a $\mathrm{Na}$ wedge as a function of angular opening $2 \varphi$ (degrees) for linear densities $n=10$ and $15 \AA^{-1}$. For $n=15 \AA^{-1}$, wedge configurations within the thin vertical segments are stable with respect to the infinite pancake configuration. For $n=10 \AA^{-1}$, wedge configurations to the right of the corresponding thin vertical segment are stable with respect to the infinite pancake configuration.

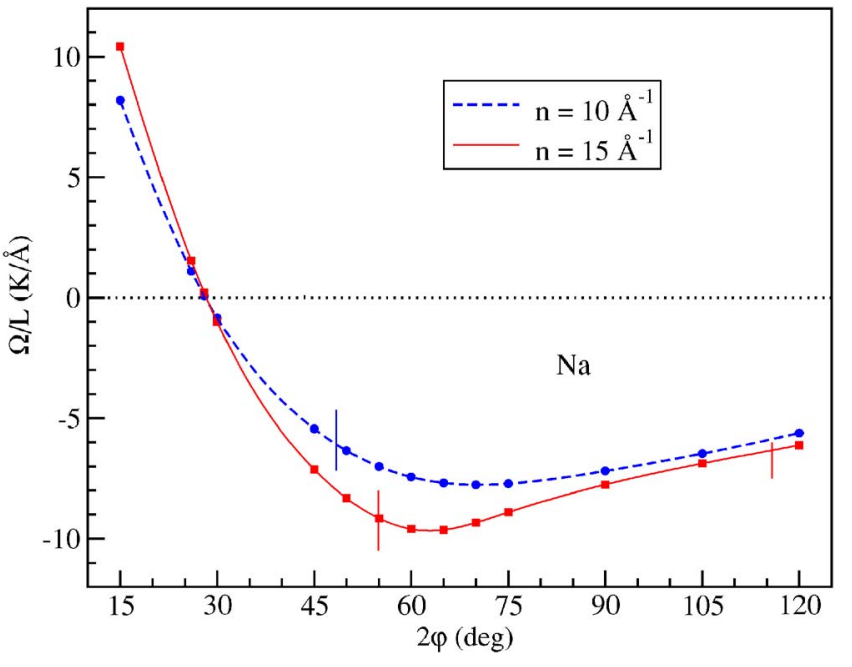

FIG. 8. (Color online) Same as Fig. 7 for the grand potential per unit length.

filling and the emptying angles, reaching a minimum at same intermediate opening. This behavior can be qualitatively appreciated from a visual standpoint, although one should avoid any quantitative formulation-such as an attempt to locate the minimum-due to the impossibility of extracting accurate geometrical parameters out of density profiles for finite samples, as discussed in, i.e., Refs. 10 and 16 in relation to the determination of the contact angle of a drop.

\section{SUMMARY}

We have presented detailed calculations of the structure and energetics of nanoscopic samples of ${ }^{4} \mathrm{He}$ in a linear wedge with two identical alkali walls. Our results indicate that for a Cs substrate, a sequence of states that includes one

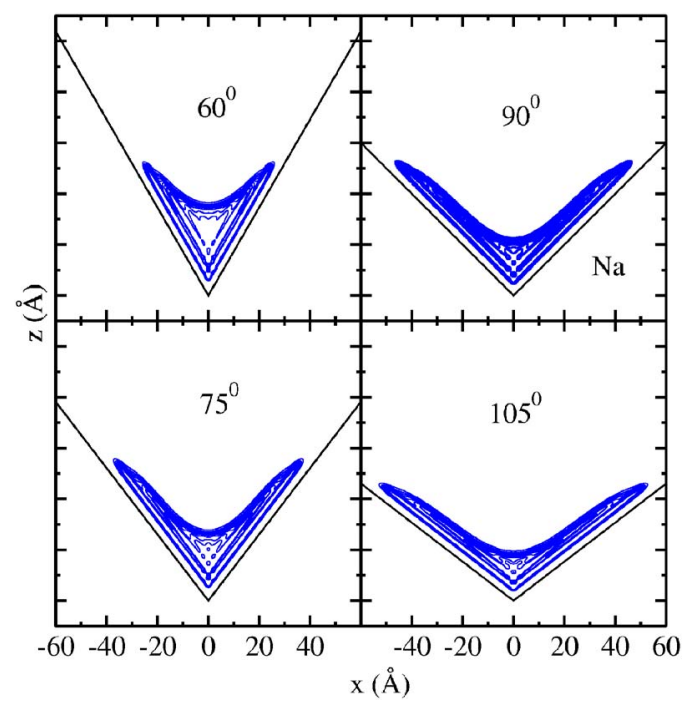

FIG. 9. (Color online) Density profiles of helium at $n=15 \AA^{-1}$ in a $\mathrm{Na}$ corner represented by thin straight lines, for angular opening $2 \varphi=60,90,105$, and $120^{\circ}$. The distance between major tick marks on the $z$ axis is $20 \AA$. Equidensity lines as in Fig. 2. 
filling transition and a threshold for-metastableconfigurations where the wedge is void of ${ }^{4} \mathrm{He}$ occurs by varying the wedge opening at zero temperature, with condensation at negative grand potential (accompanied by prefilling jumps in the linear density) as an intermediate regime. When the material is near saturation, the angle for the filling transition is stable against changes in the linear density and can be identified with the contact angle on a planar substrate. It is important to bear in mind that the experimental realization of such system, which could be done in practice by depositing the chosen alkali on a silicon surface previously patterned with microscale wedges, ${ }^{25}$ is very challenging due to the reactive character of alkalis.

The present wedge geometry can be also used as a first step to model rough alkali surfaces. Quite surprisingly, our calculations indicate that narrow Cs wedges (of nanoscopic size) are not filled by finite amounts of helium. This circumstance might be relevant in the study of the wetting behavior of ${ }^{4} \mathrm{He}$ on rough $\mathrm{Cs}$ films, and in the modeling of helium nanopuddles formed on strong-pinning surfaces. ${ }^{26}$

We have also carried out similar calculations for helium on sodium which indicate that a helium pancake originally deposited on a flat, strongly wettable adsorber like $\mathrm{Na}$, basically follows the folding of the surface with angle $2 \varphi$ and forms a deep meniscus. Last, we have shown that a theoretical search of the vanishing of the grand potential as a function of wedge opening, along with liquid-vacuum coexistence at $\mu_{0}$ and independent on the linear coverage $n$, is feasible within a DF description and unambiguously determines, from the theory point of view, the contact angle of a nanoscopic helium sample on a nonwettable substrate.

\section{ACKNOWLEDGMENTS}

We are indebted to F. Caupin, M. W. Cole, R. Estalella, H. Godfrin, G. Mistura, and A. F. G. Wyatt for stimulating discussions and helpful advice. This work was supported by grants PIP2391/00 from CONICET and PICT03-08450 from ANPCYT, Argentina, FIS2005-01414 from DGI, Spain (FEDER), and 2005SGR-00343 from Generalitat of Catalunya. F.A. acknowledges funding from CESCA-CEPBA, Barcelona, through the program HPC-Europa Transnational Access, and Project No. CPDA033545 of Padova University.
${ }^{1}$ C. Rascón and A. O. Parry, J. Chem. Phys. 112, 5175 (2000).

${ }^{2}$ C. Rascón and A. O. Parry, Nature (London) 407, 986 (2000).

${ }^{3}$ J. R. Henderson, Phys. Rev. E 69, 061613 (2004).

${ }^{4}$ E. Cheng and M. W. Cole, Phys. Rev. B 41, 9650 (1990).

${ }^{5}$ M. Napiórkowski, W. Koch, and S. Dietrich, Phys. Rev. A 45, 5760 (1992).

${ }^{6}$ P. Concus and R. Finn, Phys. Fluids 10, 39 (1997).

${ }^{7}$ K. Rejmer, S. Dietrich, and M. Napiorkowski, Phys. Rev. E 60, 4027 (1999).

${ }^{8}$ E. H. Hauge, Phys. Rev. A 46, 4994 (1992).

${ }^{9}$ L. Bruschi, A. Carlin, and G. Mistura, Phys. Rev. Lett. 89, 166101 (2002).

${ }^{10}$ M. Barranco, M. Guilleumas, E. S. Hernández, R. Mayol, M. Pi, and L. Szybisz, Phys. Rev. B 68, 024515 (2003).

${ }^{11}$ F. Dalfovo, A. Lastri, L. Pricaupenko, S. Stringari, and J. Treiner, Phys. Rev. B 52, 1193 (1995).

${ }^{12}$ A. Chizmeshya, M. W. Cole, and E. Zaremba, J. Low Temp. Phys. 110, 677 (1998).

${ }^{13}$ L. W. Bruch, M. W. Cole, and E. Zaremba, Physical Adsorption: Forces and Phenomena (Oxford Science Publications, New York, 1997).

${ }^{14}$ M. W. Cole, F. Ancilotto, and S. M. Gatica, J. Low Temp. Phys.
138, 195 (2005)

${ }^{15}$ M. Frigo and S. G. Johnson, Proc. IEEE 93(2), 216 (2005).

${ }^{16}$ F. Ancilotto, A. M. Sartori, and F. Toigo, Phys. Rev. B 58, 5085 (1998).

${ }^{17}$ F. Ancilotto, F. Faccin, and F. Toigo, Phys. Rev. B 62, 17035 (2000).

${ }^{18}$ E. Rolley and C. Guthmann, J. Low Temp. Phys. 108, 1 (1997).

${ }^{19}$ D. Ross, J. E. Rutledge, and P. Taborek, Science 278, 664 (1997);

J. E. Rutledge, D. Ross, and P. Taborek, J. Low Temp. Phys. 113, 811 (1998).

${ }^{20}$ F. Ancilotto, F. Faccin, and F. Toigo, Phys. Rev. B 62, 17035 (2000).

${ }^{21}$ J. Klier, P. Stefanyi, and A. F. G. Wyatt, Phys. Rev. Lett. 75, 3709 (1995).

${ }^{22}$ We recall that in the macroscopic limit, the conditions $\Omega / L=0$, $\Omega / L$ independent of $n$, and $\mu=\mu_{0}$, happen simultaneously.

${ }^{23}$ S. M. Gatica, M. M. Calbi, and M. W. Cole, Phys. Rev. E 59, 4484 (1999).

${ }^{24}$ L. Szybisz and I. Urrutia, Phys. Rev. E 66, 051201 (2002).

${ }^{25} \mathrm{G}$. Mistura (private communication).

${ }^{26}$ J. Klier, P. Leiderer, D. Reinelt, and A. F. G. Wyatt, Phys. Rev. B 72, 245410 (2005). 